\title{
SIMPLE OPTOELECTRONIC EXTEROCEPTIVE SENSOR FOR THE CONTROL OF THE DYNAMIC EQUILIBRIUM OF A WALKING ROBOT
}

\author{
ERIK KRÁL \\ Tomas Bata University in Zlin, Faculty of Applied Informatics \\ Nad Stránèmi 4511, CZ-76005 Zlin, Czech Republic
}

\begin{abstract}
Simple optoelectronic exteroceptive sensor for controlling and learning the dynamical equilibrium of a walking robot is presented. Sensor consists of the digital camera and structured light source, for example laser diode module with Diffractive Optical Element. Digital camera captures structured configuration of light spots projected on a surface in front of a robot. There are two variants, the light source position is fixed to the digital camera and there is no reference object at scene. In these case only medial and lateral tilt to plane in front of the robot is estimated and second variant when only light source of the robot is fixed to any part of robot and camera is placed somewhere else. In these case multi-degrees-of-freedom information can be estimated. The image information from the digital camera is input for the control of a dynamical equilibrium of a walking robot.
\end{abstract}

\section{Introduction}

This article proposes simple optoelectronic exteroceptive sensor for the control of the dynamical equilibrium of a walking robot mainly for the indoor environments. It is not supposed as a main control device, but it is means as an extension for optimal motion on a planar surface. There are two versions, first the camera is fixed to the light source and placed to the robot oriented toward, then the medial and lateral tilt is estimated as the input information for the control of a dynamic equilibrium of a walking robot depicted in Figure 1. Additionally, the second camera, placed apart from the robot can be used if there is a reference object with two pair of parallel lines [1]. In this case, multidegrees-of-freedom information can be used for the control of a dynamical equilibrium of a walking robot, depicted in Figure 2. 


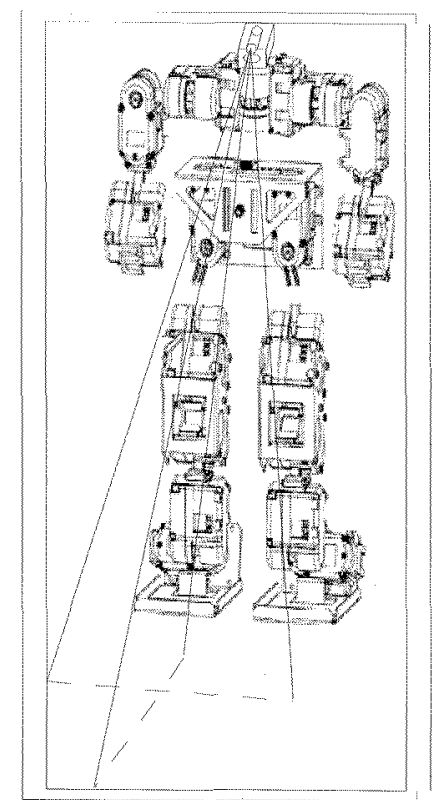

Figure 1.Walking robot with mounted optoelectronic exteroceptive sensor.

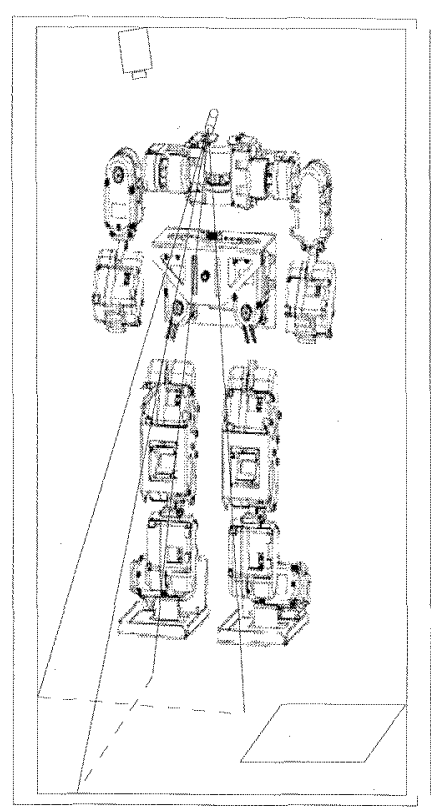

Figure 2. Second camera and reference rectangle

\section{Methods}

The main part of the image processing is done using the algorithm for the analysis of the image information for the accuracy of the positioning of the laser diode's light spots [3], depicted in Figure 3 which creates the input information for the control of the walking robot. The laser light spots positions are determined using basic matching algorithm. Using 2-D Gauss distribution precise position of the center of the lasers light spot is estimated. The camera system distortion has to be considered and is eliminated by the application of the calibration camera model [4] and [5]. Perspective distortion is removed using two parallel lines in a reference object [6]. A projective transformation can map ideal points to finite points. Under an affine transformation the ideal points remain at infinity [6].

\section{Algorithm components}

In Figure 3 you can see the example of the pattern consist of four points A, B, C, $D$ projected with four laser beams from one center with the uniform inter-beam angle $\sigma$. These laser beams creates pyramid. In case, that there is any reference object with know pair of parallel lines, perspective distortion could be removed using the two parallel lines in reference object [3]. It means that there should be 
another reference pattern, square or rectangle, with known parallel lines. In this study, only the pattern with four points is described. The main reason is that it could be easily built with four low-cost laser diodes mounted in desired configuration. This algorithm is designed to be used in low cost embedded hardware like FPGA or microcontroller.

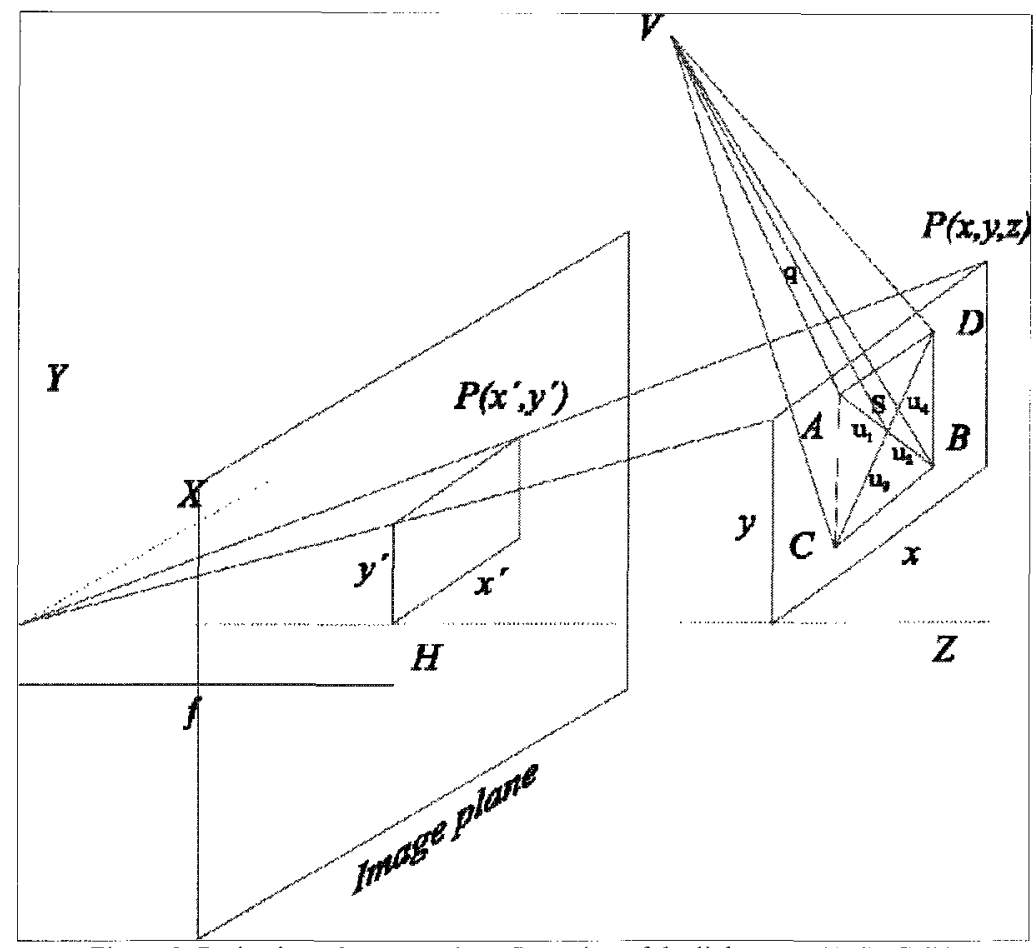

Figure 3. Projection of structured configuration of the light spots (A, B, C, D)

Algorithm has these basic steps

1) Estimation of the suspicious areas

2) Finding the suitable areas combination

3) Camera distortion elimination

4) Estimation of the centre of the light spot using 2-D Gauss distribution

5) Estimation of the medial and lateral tilt or of the multi-DoF information.

The matching algorithm uses the pattern with the discrete Gauss distribution. Only areas with a sudden change of brightness are tested. The suspicious areas are the areas which match the pattern and are small enough to be considered as 
light spots. First step is the affine rectification. A projective transformation can map ideal points to finite points. Under an affine transformation the ideal points remain at infinity [6]. Angles $\beta_{1}, \beta_{2}$ of the rotations of the pyramid axis around pyramid's base diagonals are computed from ratio of length of $u_{l}, u_{2}$ respective $u_{3}, u_{4}$ :

$$
\begin{aligned}
& k_{1}=\frac{u_{1}}{u_{3}} k_{2}=\frac{u_{2}}{u_{4}} \\
& \beta_{1}=a \cos \frac{\sqrt{\left(1+k_{1}\right)^{2}} \sin \sigma}{\sqrt{1+k_{1}^{2}-2 k_{1}} \cos (2 \sigma)} \\
& \beta_{2}=a \cos \frac{\sqrt{\left(1+k_{2}\right)^{2}} \sin \sigma}{\sqrt{1+k_{2}^{2}-2 k_{2} \cos (2 \sigma)}}
\end{aligned}
$$

Where $\sigma$ is the light source inter-beam angle and $u_{1} \geq u_{3} ; u_{2} \geq u_{4}$. As the affine transformation preserve ratio of lengths on collinear lines, the ratio of $k_{l}$ and $k_{2}$ should be equal. Then, the expected positions of light's beams with known angles are constructed. These positions are compared with measured position and parameters of the inverse affine transformation are computed.

As the affine transformation preserve ratio of lengths on collinear lines, the ratio of $k_{1}$ and $k_{2}$ should be the same. Then, the expected positions of light's beams with known angles are constructed. These positions are compared with measured position and parameters of inverse affine transformation are computed. Combinations with low affine transformation error and with the low difference of the ratio $k_{1}$ and $k_{2}$ are chosen. The best combination is finally estimated according to its brightness and level of matching to the pattern.

By solving system of equations (2) for 3 and more corresponding points, we can find matrix $T$, which can be easily transformed to the affine transformation matrix $T_{A}$.

$$
\begin{gathered}
{\left[\begin{array}{l}
x^{\prime} \\
y^{\prime} \\
1
\end{array}\right]=T\left[\begin{array}{l}
x \\
y \\
1
\end{array}\right] T=\left[\begin{array}{lll}
a & b & c \\
d & e & f \\
g & h & i
\end{array}\right]} \\
a x+b y+c-g x^{\prime} x-h x^{\prime} y-i x^{\prime}=0 \\
d x+e y+f-g y^{\prime} x-h y^{\prime} y-i y^{\prime}=0
\end{gathered}
$$




$$
\left[\begin{array}{ccccccccc}
x_{1} & y_{1} & 1 & 0 & 0 & 0 & -x_{1}^{\prime} x_{1} & -x_{1}^{\prime} y_{1} & -x_{1}^{\prime} \\
0 & 0 & 0 & x_{1} & y_{1} & 1 & -y_{1}^{\prime} x_{1} & -y_{1}^{\prime} y_{1} & -y_{1}^{\prime} \\
\vdots & \vdots & \vdots & \vdots & \vdots & \vdots & \vdots & \vdots & \vdots \\
x_{n} & y_{n} & 1 & 0 & 0 & 0 & -x_{n}^{\prime} x_{n} & -y_{n}^{\prime} x_{n} & -x_{n}^{\prime} \\
0 & 0 & 0 & x_{n} & y_{n} & 1 & -y_{n}^{\prime} x_{n} & -y_{n}^{\prime} y_{n} & -y_{n}^{\prime}
\end{array}\right]=\left[\begin{array}{c}
a \\
b \\
c \\
\vdots \\
i
\end{array}\right]
$$

The radial distortion can be approximated using the expression (6) and tangential distortion is often describes as expression (7)[5]

$$
\begin{gathered}
{\left[\begin{array}{l}
\tilde{u}_{i} \\
\tilde{v}_{i}
\end{array}\right]=\left[\begin{array}{c}
\frac{x}{z} \\
\frac{y}{z}
\end{array}\right]} \\
{\left[\begin{array}{l}
\delta u_{i}^{(r)} \\
\delta v_{i}^{(r)}
\end{array}\right]=\left[\begin{array}{l}
\tilde{u}_{i}\left(k_{1} r_{i}^{2}+k_{2} r_{i}^{4}+\ldots\right) \\
\widetilde{v}_{i}\left(k_{1} r_{i}^{2}+k_{2} r_{i}^{4}+\ldots\right)
\end{array}\right] r_{i}=\sqrt{\tilde{u}_{i}^{2}+\widetilde{v}_{i}^{2}}} \\
{\left[\begin{array}{l}
\delta u_{i}^{(i)} \\
\delta v_{i}^{(r)}
\end{array}\right]=\left[\begin{array}{l}
2 p_{1} \tilde{u}_{i} \widetilde{v}_{i}+p_{2}\left(r_{i}^{2}+2 \widetilde{u}_{i}^{2}\right) \\
p_{2}\left(r_{i}^{2}+2 \widetilde{v}_{i}^{2}\right)+2 p_{2} \widetilde{u}_{i} \widetilde{v}_{i}
\end{array}\right] r_{i}=\sqrt{\tilde{u}_{i}^{2}+\tilde{v}_{i}^{2}}}
\end{gathered}
$$

A camera model for distortion calibration is derived by combining the CCD camera projection model (5) with the correction for the radial and tangential distortion components.

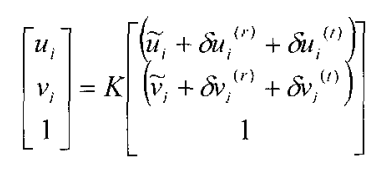

Precise positions of the centers of the laser light spots are estimated using 2-D Gauss distribution approximation [3]. And we considered the centre of 2-D Gauss distribution approximation as the centre of the laser light spot. In case, there is reference object, positions of the centers of the laser light spots can be transformed into the three rotations and three translations [7].

As a final step, the information about the medial and lateral tilt or of the multiDoF information can be used as input information to the neural network that provides control of the dynamic equilibrium of a walking robot.

\section{Implementation}

Evaluating algorithms are implemented in MATLAB and executive algorithms are implemented in the Field Programmable Gate Array (FPGA) with the Very 
High Density Hardware Description Language (VHDL).

\section{Conclusion}

This article propose simple optoelectronic sensor for the control of the dynamical equilibrium of a walking robot mainly in indoor environments. The medial and lateral tilt or multi-degrees-of-freedom information can be used for the control of the walking robot or for the learning of walking robot.

\section{Acknowledgments}

The financial support from the grant MSM7088352102, ,Modeling and Control of Processing Procedures of Natural and Synthetic Polymers" is gratefully acknowledged.

\section{References}

1. E. Král, Preliminary Study of Construction of Optoelectronic Control Device for Object Modeling in 3D Virtual Environment, Ostrava: International Workshop Control and Information Technology (2007) ISBN 978-80-8073-805-1

2. M. Kvasnica, Six DoF Sensory system for the force-torque control.of walking humanoid. Proceedings of the 11th International Conference on Climbing and Walking Robots and the Support Technologies for Mobile Machines, Coimbra, Portugal, 08 - 10 September 2008.

3. E. Král, The analysis of the image information for the accuracy of the positioning of the multicomponent sensory system's laser diode's light spots. Strbské Pleso: 8th International Carpathia Control Conference, (2007), ISBN 978-80-8073-805-1

4. C. Mei, 2006. Camera Calibration Toolbox for Matlab $<$ http://www.vision.caltech.edu/bouguetj/calib_doc/ >

5. R. Tsai, An Efficient and Accurate Camera Calibration Technique for 3D Machine Vision, Proceedings of IEEE Conference on Computer Vision and Pattern Recognition, Miami Beach, FL, pp. 364-374 (1986)

6. R. Hartley and A. Zisserman, Multiple View Geometry in Computer Vision. Second Edition, Cambridge University Press (2004), ISBN 0521540518

7. M. Kvasnica. Algorithm for Computing of Information about Six-DOF Motion in 3-D Space Sampled by 2-D CCD Array, The paper from Invited Session by Kvasnica M.: "Robotics, Sensory Systems for Robotics and for Human-Machine Interface", Proceedings of the World Multi-Conference SCI '2001-ISAS, Vol.XV, Industrial Systems, Part II, Orlando, Florida, July 2001, USA. 